\title{
AMENDMENTS
}

\section{Author Correction: A dynamic three-step mechanism drives the HIV-1 pre-fusion reaction}

Maro Iliopoulou, Rory Nolan, Luis Alvarez iD, Yasunori Watanabe, Charles A. Coomer, G. Maria Jakobsdottir, Thomas A. Bowden (D) and Sergi Padilla-Parra (D)

Correction to: Nature Structural \& Molecular Biology https://doi.org/10.1038/s41594-018-0113-x, published online 27 August 2018.

In the version of this article initially published, the label above the top right plot in Fig. 3b (HXB2-Alexa Fluor 488) was incorrect. The correct label is 'HXB2-Alexa Fluor 405'. The error has been corrected in the HTML and PDF versions of the article.

Published online: 9 May 2019

https://doi.org/10.1038/s41594-019-0244-8

\section{Publisher Correction: RNA modification: Getting a hold on cytosine methylation in mRNA}

Lukas Trixl and Alexandra Lusser (D)

Correction to: Nature Structural \& Molecular Biology https://doi.org/10.1038/s41594-019-0217-y, published online 6 May 2019.

In the version of this article initially published, the author order (Liu, J., Huang, T., Chen, W., Gu, N. \& Zhang, R.) for the linked Resource (ref. 7) was incorrect, and all named citations of that reference in the text ("Liu et al.") were incorrect. The correct author order is "Huang, T., Chen, W., Liu, J., Gu, N. \& Zhang, R." and all named citations should reflect that ("Huang et al."). The error has been corrected in the HTML and PDF versions of the article.

Published online: 14 May 2019

https://doi.org/10.1038/s41594-019-0247-5

\section{Publisher Correction: Tauopathies: Protein shapes at the core of chronic traumatic encephalopathy}

Yann Fichou and Songi Han

Correction to: Nature Structural \& Molecular Biology https://doi.org/10.1038/s41594-019-0221-2, published online 22 April 2019.

In the version of this article initially published, the Acknowledgements section was missing. The Acknowledgements are as follows: "Y.F. is supported by the National Institute of Aging of the US National Institutes of Health (NIH) through grant R01AG05605. S.H. acknowledges support of that NIH grant R01AG05605 and the Tau Consortium of the Rainwater Charitable Foundation." The error has been corrected in the HTML and PDF versions of the article. 